\title{
Año Internacional de la Luz: Aniversario de hitos importantes en la historia de la ciencia de la luz
}

María Cristina Pineda de Carías.

\section{Resumen}

En el marco del 2015 Año Internacional de la Luz, este trabajo presenta un repaso de hitos importantes en la historia de la ciencia de la luz cuyo aniversario se celebra también este año. En un estudio histórico bibliográfico que abarca un período de mil años, se documenta: 1) la labor sobre la óptica de Ibn Alhacén en 1015; 2) la noción del carácter ondulatorio de la luz propuesta por Fresnel en 1815; 3) la teoría electromagnética de propagación de la luz formulada por Maxwell en 1865; 4) la teoría de Einstein del efecto fotoeléctrico en 1905; 5) la incorporación de la luz en la cosmología mediante la relatividad general de Einstein en 1915; 6) el descubrimiento del fondo de microondas del cosmos por Penzias y Wilson en $1965 ; 7)$ los logros alcanzados por Kao en la transmisión de luz por fibras para la comunicación óptica, en 1965. Todos estos descubrimientos científicos se ponen en contexto para destacar, como lo señala la resolución de las Naciones Unidas, que la celebración del Año Internacional de la Luz ha sido una magnífica oportunidad para reconocer la importancia de la enseñanza de las ciencias en nuestro país.

Palabras clave: 2015 Año Internacional de la luz; hitos en la historia de la ciencia de la luz; Alhacén, Fresnel, Maxwell, Einstein, Penzias y Wilson, Kao

\section{Abstract}

Within the frame of 2015 International Year of Light, this paper presents an overview of important milestones in the history of the science of light whose anniversary is celebrated this year. In a historic and bibliographic study that covers one thousand years, we document: 1) the works on optics by Ibn Al-Haytham in 1015; 2) the notion of light as a wave proposed by Fresnel in 1815;3) the electromagnetic theory of light propagation proposed by Maxwell in 1865; 4) Einstein's theory of the photoelectric effect in 1905; 5) the embedding of the light in cosmology through Einstein's general relativity in $1915 ; 6$ ) the discovery of the cosmic microwave background by Penzias and Wilson in 1965; 7) Kao's achievements concerning the transmission of light in 
fiber for optical communications in 1965. All these scientific discoveries are placed in the context of the United Nations Resolution, highlighting that the International Year of Light celebrations provide an opportunity to recognize the importance of teaching sciences in our country.

Keywords: 2015 International Year of Light; milestones in the history of the science of light; Al-Haytham, Fresnel, Maxwell, Einstein, Penzias and Wilson, Kao.

María Cristina Pineda de Carías, (mcpinedacarias@gmail.com),Decana de la Facultad de Ciencias Espaciales, Universidad Nacional Autónoma de Honduras - UNAH. 
La Organización de las Naciones Unidas en su Asamblea General del 20 de diciembre de 2013, mediante Resolución 68/221 decidió proclamar el año 2015 Año Internacional de la Luz y las Tecnologías Basadas en la Luz (2014). Esta resolución fue aprobada por la Asamblea General:

"Reconociendo la importancia de la luz y las tecnologías basadas en la luz para la vida de los ciudadanos del mundo y para el desarrollo futuro de la sociedad mundial en muchos niveles,

Destacando que el aumento de la conciencia mundial y un fortalecimiento de la enseñanza de la ciencia y las tecnologías de la luz son esenciales para abordar retos tales como el desarrollo sostenible, la energía y la salud de las comunidades, así como para mejorar la calidad de vida en los países desarrollados y en desarrollo..."

En Honduras, motivados por esta Resolución, el Departamento de Astronomía y Astrofísica de la Facultad de Ciencias Espaciales de la Universidad Nacional Autónoma de Honduras, organizó el Nodo Nacional del Año Internacional de la Luz 2015 encargado de realizar una serie de actividades para llamar la atención de la población acerca de cómo las tecnologías de la luz han influido de manera determinante en nuestras vidas cotidianas y en una mejora de la calidad de vida. El lanzamiento del Nodo Nacional incluyó una conferencia inaugural la cual ha sido la base para la elaboración de este documento.

El objetivo de este trabajo ha sido hacer un recorrido histórico por siete importantes hitos de la luz que conmemoran su aniversario en el año 2015. Colocados en una línea de tiempo de aproximadamente un milenio, como producto de una revisión bibliográfica se presentan las contribuciones de reconocidos personajes de la ciencia que en señalados años marcaron la historia de la ciencia y las tecnologías de la luz. Por períodos de tiempo, años, personas y sus aportes, los hitos presentados son los siguientes:

- Hace 1,000 años, 1015: Ibn Alhacén, por su labor sobre la óptica.

- Haciendo un salto de 800 años después, 1815: Fresnel, por la noción del carácter ondulatorio de la luz.

- 50 años después, 1865: Maxwell, por la teoría electromagnética de propagación de la luz.

- 40 años después, 1905: Einstein, por la teoría del efecto fotoeléctrico. 
- 10 años después,1915: nuevamente Einstein, por la incorporación de la luz en la cosmología mediante la relatividad general.

- 50 años después, en 1965: primero Penzias y Wilson, por el descubrimiento del fondo de microondas del cosmos.

- Para terminar, en ese mismo año de 1965: con Kao, por los logros alcanzados en la transmisión de la luz por fibras para la comunicación óptica.

Con este recorrido se trata de revisar nuestro entendimiento de la luz en el milenio comprendido de 1015 a 2015, como lo vivimos, y como hoy se reconoce su importancia para la vida de los ciudadanos del mundo, y para el desarrollo futuro de la sociedad mundial en muchos niveles.

\section{5: IBN ALHACÉN}

Abū 'Alī al-Hasan ibn al-Hasan ibn al-Haylam nació en Basora, actual Irak, en el año 965 y murió en El Cairo, Egipto en el año 1040. Conocido en español como Alhacén, fue un matemático, físico y astrónomo musulmán (Wikipedia, 2015).

Estudios bio-bibliográficos de Alhacén revelan lo muy extensa que es la muestra de títulos de las obras que se le atribuyen, y de la que se ha conservado un porcentaje apreciable hasta nuestros días (González-Cano, 2015). De excepcional se califica su trabajo y aportes a la ciencia, la filosofía y, en particular a la óptica y a las teorías sobre el mecanismo de la visión en los seres vivos (Calvo, 2015).

Alhacén conoció y asimiló de manera casi completa los textos de tradición griega sobre Óptica, Matemáticas, Astronomía, Medicina y otras áreas. Se ocupó de traducir al árabe y producir manuscritos de los Elementos de Euclides y el Almagesto de Ptolomeo.

Se le considera como el padre de la Óptica, porque entre sus experimentos y estudios incluyó una teoría de la luz y una teoría de la visión. El suyo fue, no solo un trabajo integrador de los conocimientos de su tiempo, sino que fue trascendental por los aportes de su propio talento. Como en el caso de Ptolomeo, la Óptica de Alhacén: Kitab al-Monazir, fue escrita en siete libros que él mismo describe así:

"Dividimos esta obra en siete libros. En el primero nos ocupamos de la visión de forma general. En el libro II detallamos las propiedades visibles, 
sus causas y el modo en que se perciben. En el libro III mostramos los errores de la vista en lo que percibe directamente, y sus causas. En el libro IV tratamos de la percepción visual por reflexión de los cuerpos lisos. En el libro $V$ mostramos la posición de las imágenes, es decir, las formas vistas dentro de los cuerpos lisos. En el libro VI nos ocupamos de los errores de la visión en lo que percibe por reflexión, y sus causas. En el libro VII estudiamos la percepción visual por refracción a través de cuerpos transparentes, cuya transparencia difiere de la del aire. Con el final de este libro concluye la obra (Sabra 1989 en González-Cano 2015, pág. 5).

Antes de la edición de la Óptica de Alhacén se discutían dos teorías alternativas de la visión. Una, la extromísiva, que afirmaba que ciertas formas de radiación se emitían desde los ojos en forma cónica sobre el objeto que se estaba viendo. El sorprendente haz de rayos que llegaba al objeto permitía percibir aspectos tales como el color, la forma y el tamaño de un objeto.

La otra teoría, intromisiva, era la que sostenía que los rayos luminosos eran transmitidos a los ojos permitiendo la percepción de un objeto o su entorno. Como una demostración de esta teoría, a principios del siglo XI, en 1015, Alhacén realizó un experimento crítico que refiere lo que ocurre después que un observador ha mirado directamente al sol: "al cerrar los ojos, el observador percibe una post-imagen del sol, estando aquel con los párpados cerrados. La post-imagen se desvanece gradualmente hasta desaparecer. En sus explicaciones, (Alhacén) argumentó que este fenómeno no podía ser el resultado de 'algo' emitido desde dentro del ojo (lo que los defensores de la teoría de la extramisión llamaban 'rayo visual'). Por el contrario, el fenómeno debía ser causado por una fuente o estímulo externo." (Calvo, 2015, pág. 34). Al repetir el experimento con el sol y otras fuentes brillantes vistas a través de un espejo o reflejado en un segundo objeto, los resultados fueron similares.

Con sus trabajos y experimentos, Alhacén dio la primera explicación de la visión y demostró que la luz es reflejada desde cada punto de un objeto hacia el ojo del observador.

Basándose en el hecho de que el color con que se ven los objetos está en consonancia con la luz que los ilumina, pensó que la luz debía intervenir en la visión, más precisamente que debía ser considerada como una entidad independiente del objeto y del ojo que hace de intermediario de la visión. 
Alhacén fue el primero en dar una interpretación clara del funcionamiento de la cámara oscura y planteó un modelo de visión según el cual esta consistía en la formación de una imagen óptica en el interior del ojo que funcionaba de forma semejante al modelo de su cámara oscura.

Él elaboró su propia teoría postulando que un objeto observado emite rayos de luz desde cada punto de su superficie que se desplazan a los ojos del espectador. Según esta teoría, el objeto observado no es considerado como un objeto completo, sino como la suma de una cantidad infinita de puntos que juntos componen la totalidad del objeto del que se proyectan los rayos de luz.

En su libro de Óptica Alhacén utilizó conceptos novedosos afirmando que hay dos tipos de luz, la luz principal y la luz secundaria, siendo la luz principal la más intensa de la luz que procede de cuerpos auto luminosos, y la luz secundaria que procede de los objetos que accidentalmente la reciben y la reflejan de los cuerpos auto luminosos. Tanto la luz primaria como la secundaria viajaban en línea recta.

Su libro de Óptica ha sido considerado como uno de los más influyentes, por eso se ha considerado un hito en la historia de la Ciencia de la Luz.

\section{5: AUGUSTÍN FRESNEL}

En noviembre de 1801, julio de 1802 y noviembre de 1803, Thomas Young (17731829) leyó artículos ante la Royal Society de Londres exaltando la teoría ondulatoria de la luz, y añadiendo a ella un nuevo concepto fundamental, el llamado principio de interferencia que dice que "cuando dos ondulaciones de diferentes orígenes coinciden perfectamente en dirección o casi coinciden, su efecto conjunto es una combinación de los movimientos que pertenecen a cada una". Los artículos de Young fueron severamente atacados, llegando a decirse que estaban desprovistos de mérito (Hecht \& Zajac, 1986).

Esta respuesta al trabajo de Young no fue más que el arrastre de la profunda controversia entre las dos teorías sobre la naturaleza de la luz, iniciada desde finales del siglo XVII. Una de las teorías, la teoría corpuscular, establecía que la luz estaba formada de partículas llamadas corpúsculos que se propagaban en línea recta y atravesaban medios transparentes. Fue defendida por Isaac Newton (1642-1727) por lo que la teoría gozó de mucho prestigio en el siglo XVIII. La otra teoría, la teoría ondulatoria, defendida por Cristian Huygens (1629-1695) establecía que la luz era una onda que se propagaba en un medio transparente llamado el éter (Alonso Sánchez, 2015). 
Ajeno a los trabajos de Young, en 1815 Augustin Fresnel publicó la "Premier Mémoire sur la Diffraction de la Lumière" (Primera Memoria sobre la difracción de la luz) y "Théorie de la Lumière" (Teoría de la Luz) reviviendo en Francia, de manera brillante y con gran rigor matemático la teoría ondulatoria de la luz (Beléndez, 2015). Augustin-Jean Fresnel fue un físico e ingeniero francés que estudio el comportamiento de la luz tanto teórica como experimentalmente. Nació en Broglie el 10 de mayo de 1788, y murió el 14 de julio de 1827 (Wikipedia, 2015).

En 1818, Fresnel presentó su teoría sobre la difracción ondulatoria de la luz a un certamen en la Academia de Ciencias francesa (Bachiller, 2015). Simeón Poisson (1781-1840), físico matemático seguidor de la teoría corpuscular de Newton fue miembro del jurado. Poisson estudió en detalle las ecuaciones de Fresnel llegando a deducir matemáticamente, a partir de ellas que, si se iluminaba un disco opaco con luz monocromática, en el centro de la sombra del disco debía formarse un punto brillante. Poisson consideró que esta era una conclusión absurda y que la teoría de Fresnel era errónea.

El presidente del jurado de este certamen, el matemático, físico y astrónomo francés François Jean Dominique Arago (1786-1853), decidió llevar a cabo el experimento preparando un montaje cuidadoso para poder observar el punto luminoso predicho por Poisson. "Gracias a este espectacular experimento, Fresnel no solo ganó el certamen de la Academia, sino que dejó plenamente convencidos a los científicos de la época de que la luz era realmente una onda." El famoso experimento se conoce ahora como el "punto de Arago".

Con sus trabajos, Fresnel sintetizó los conceptos de la teoría ondulatoria de Huygens y el principio de interferencia. El modo de propagación de una onda primaria lo miró como una sucesión de pequeñas ondas secundarias, que se superponían e interferían para reformar la onda primaria en su avance. Supuso que estas ondas eran longitudinales en analogía con las ondas de sonido del aire. Fresnel pudo calcular los patrones de difracción generados en varios obstáculos y aperturas y explicó satisfactoriamente la propagación rectilínea en medios isotrópicos homogéneos, eliminando así la objeción principal de Newton para la teoría ondulatoria.

Como era de esperar, Fresnel fue informado de la prioridad de Young sobre el principio de interferencia. Entonces Fresnel trató de encontrarse con Young, y ambos se hicieron amigos. Además, Arago fue uno de los primeros en convertirse a la teoría ondulatoria de Fresnel, y también se hicieron amigos y colaboradores. 
Young, Arago y Fresnel lucharon con varios problemas. Young sugirió que la vibración del éter podía ser transversal. La luz era entonces simplemente una manifestación de dos vibraciones ortogonales del éter, transversales a la dirección del rayo. Aquí es donde Fresnel comenzó a desarrollar una descripción mecánica de las oscilaciones del éter, las que lo llevaron a sus famosas fórmulas para la amplitud de la luz reflejada y transmitida.

Después de este hito en la historia de la ciencia de la luz, muy pocos eran los partidarios de la teoría corpuscular.

\section{5: JAMES CLERK MAXWELL}

El tercer hito en la historia de la ciencia de la luz, ocurrió en el campo de la electricidad y el magnetismo. En 1865 Maxwell, mediante un conjunto de ecuaciones demostró que la electricidad, el magnetismo y hasta la luz, son manifestaciones del mismo fenómeno: el campo electromagnético.

James Clerk Maxwell, nació el 13 de junio de 1831 en Edimburgo, Inglaterra, y murió el 5 de noviembre de 1879 en Cambridge, Inglaterra. Fue un físico escocés conocido principalmente por haber desarrollado la teoríaelectromagnética clásica, sintetizando todas las anteriores observaciones, experimentos y leyes sobre electricidad, magnetismo y aún óptica en una teoría consistente (Wikipedia, 2015).

El 1 de enero de 1865, la Real Sociedad de Londres publicó el famoso trabajo de Maxwell sobre Una Teoría Dinámica del Campo Electromagnético (Maxwell, 1865).

En este artículo Maxwell explica: "La teoría que yo propongo se llama teoría del Campo Electromagnético, porque tiene que ver con el espacio en el vecindario de los cuerpos eléctricos y magnéticos, y se llama Teoría Dinámica, porque asume que en ese espacio hay materia en movimiento, por la cual los fenómenos electromagnéticos observados son producidos. El campo electromagnético es esa parte del espacio la cual contiene y rodea los cuerpos en condiciones eléctricas y magnéticas." (Maxwell, pág. 460).

Maxwell resumió brillantemente e incluso amplió todo el conocimiento empírico que se tenía sobre el tema hasta entonces mediante un simple conjunto de ecuaciones matemáticas.

En una síntesis notablemente sucinta y bellamente simétrica como suele decirse, Maxwell pudo demostrar teóricamente, que el medio electromagnético se podía propagar como una onda transversal en el éter luminífero. 
Resolviendo la velocidad de la onda, llegó a una expresión en términos de las propiedades eléctricas y magnéticas del medio (véase ecuación 1):

$$
c=1 / \sqrt{\varepsilon_{0} \mu_{0}}
$$

Ecuación (1)

donde $\varepsilon_{0}$ es la constante eléctrica de permitividad, y $\mu 0$ la constante magnética de permeabilidad. Después de sustituir los valores conocidos y empíricamente determinados de estas cantidades, obtuvo un resultado numérico igual a la velocidad medida de la luz, $c$.

La conclusión era inevitable: la luz era "una perturbación electromagnética en forma de ondas propagadas a través del éter".

En un conjunto de originalmente 20 ecuaciones, que luego fue reducido a 4 ecuaciones, Maxwell logró integrar y describir de manera completa los fenómenos electromagnéticos.

La primera ecuación de Maxwell, llamada la Ley de Gauss para la electricidad, establece que el flujo del campo eléctrico $\overrightarrow{\boldsymbol{E}}$ a través de cualquier superficie cerrada es igual al inverso de la permitividad eléctrica $\left(1 / \varepsilon_{0}\right)$ multiplicada por la carga neta dentro de la superficie (véase ecuación 2). Esta ley describe como las líneas de campo eléctrico divergen de una carga positiva y convergen sobre una carga negativa (Tipler \& Mosca, 2008).

$$
\oint_{S} \overrightarrow{\boldsymbol{E}} \cdot d \overrightarrow{\boldsymbol{s}}=\frac{q}{s_{0}}
$$

Ecuación (2)

Experimentos clave para demostrar esta Ley fueron:

- Cargas iguales se repelen y cargas diferentes se atraen, variando esto según el inverso del cuadrado de la distancia (Ley de Coulomb).

- Una carga aplicada a un conductor aislado se mueve a su superficie exterior.

La segunda ecuación de Maxwell, la Ley de Gauss para el magnetismo, establece que el flujo del campo magnético $\overrightarrow{\boldsymbol{B}}$ a través de cualquier superficie cerrada es cero (véase ecuación 3). Esta ecuación describe la observación experimental que las líneas de campo magnético no divergen desde cualquier punto en el espacio o convergen en cualquier punto en el espacio, esto es, implica que los polos magnéticos aislados no existen (Tipler \& Mosca, 2008). 


$$
\oint_{S} \overrightarrow{\boldsymbol{B}} \cdot d \overrightarrow{\boldsymbol{s}}=0
$$

Ecuación (3)

Experimento clave para esta Ley es que:

- Es imposible crear un dipolo magnético aislado.

La tercera ecuación de Maxwell, la Ley de Faraday para la Inducción, es la que establece que la circulación del campo eléctrico $\overrightarrow{\boldsymbol{E}}$ alrededor de cualquier curva cerrada C, es igual al negativo de la rapidez de cambio del flujo del campo magnético $\overrightarrow{\boldsymbol{B}}$ a través de cualquier superficie $S$ limitada por la curva $\mathrm{C}$ (véase ecuación 4). Esta ley describe como las líneas de campo magnético rodean cualquier área a través de la cual el flujo magnético está cambiando, y relaciona el vector campo eléctrico $\overrightarrow{\boldsymbol{E}}$ con la rapidez de cambio del vector campo magnético $\overrightarrow{\boldsymbol{B}}$ (Tipler \& Mosca, 2008).

$$
\oint_{C} \overrightarrow{\boldsymbol{E}} \cdot d \overrightarrow{\boldsymbol{l}}=-\frac{d}{d t} \int_{S} \overrightarrow{\boldsymbol{B}} \cdot d \overrightarrow{\boldsymbol{s}}
$$

Ecuación (4)

Experimento clave para esta Ley es que:

- Un imán recto que se hace pasar por una espira cerrada de alambre, produce una corriente en la espira.

La cuarta ecuación de Maxwell, conocida como la Ley de Ampere, es en realidad la Ley de Ampere que Maxwell modificó para introducir la corriente de desplazamiento. Establece que la línea integral del campo magnético $\overrightarrow{\boldsymbol{B}}$ alrededor de cualquier curva cerrada $\mathrm{C}$ es igual a la constante de permeabilidad magnética $\mu 0$ multiplicada por la suma de la corriente I a través de cualquier superficie $S$ limitada por la curva y la corriente de desplazamiento $I_{d}$ a través de la misma superficie (véase ecuación 5). Esta ley describe como las líneas del campo magnético rodean un área a través de la cual una corriente o una corriente de desplazamiento están pasando (Tipler \& Mosca, 2008).

$$
\oint_{C} \vec{B} \cdot d \vec{l}=\mu_{0} I+\mu_{0} \varepsilon_{0} \int_{S} \frac{\partial E_{n}}{\partial t} d A \quad \text { Ecuación (5) }
$$


Experimentos clave de esta Ley:

- La velocidad de la luz se puede calcular mediante mediciones puramente electromagnéticas.

- Una corriente en un alambre produce un campo magnético cerca del alambre.

Maxwell introdujo los conceptos de campo y corriente de desplazamiento, y unificó los campos eléctricos y magnéticos en un solo concepto: el campo electromagnético.

Lamentablemente Maxwell murió antes de que el físico Heinrich Rudoph Hertz (1857-1894) verificara y publicara en 1888 la existencia de ondas electromagnéticas de larga longitud de onda, generándolas y detectándolas en una amplia serie de experimentos.

De esta manera Maxwell unificó la óptica con el electromagnetismo, marcando así un hito en la historia de la ciencia de la luz.

\section{5: ALBERT EINSTEIN}

El cuarto hito en la historia de la ciencia de la luz ocurrió en 1905, cuando Albert Einstein propuso la teoría del efecto fotoeléctrico.

Albert Einstein nació en Ulm, Alemania el 14 de marzo de 1879. Por diferentes razones tuvo que cambiar el lugar de su residencia viviendo primero en Múnich donde empezó su escuela primaria. Se trasladó después a Italia, y luego a Suiza donde se formó como profesor de física y matemáticas y adquirió la ciudadanía suiza. En Berna, Suiza, trabajó en una oficina de patentes y obtuvo su grado de doctor. Trabajó como profesor en varias ciudades europeas, en Berna, Zúrich, Praga y Berlín, siendo en ésta última donde en 1914 se hizo ciudadano alemán y allí permaneció hasta 1933 cuando renunció a esta ciudadanía por razones políticas y se trasladó a Estados Unidos. En Estados Unidos, fue Profesor de Física Teórica del Instituto de Estudios Avanzados de Princeton. Murió el 18 de abril de 1955, en Princeton, New Jersey (Nobel Lectures, 1967).

De la enorme producción científica de Albert Einstein, en la revista Annalen der Physik del año 1905 (Eckem, 2005) se publicaron cuatro artículos: 1) A. Einstein. AdP 17.132 (1905) [17 pp.]; 2) A. Einstein. AdP 17. 549 (1905) [12 pp]; 3) A.Einstein. AdP 17. 891 (1905) [31 pp]; 4) A. Einstein. AdP 18. 639 (1905) [3 pp]. 
El primero de estos artículos publicado el 17 de marzo de 1905 (pp.132-148), trata Sobre un punto de vista heurístico concerniente a la producción y transformación de la luz (Einstein, Zur Elektrodynamik bewegter Körper, 1905) (traducido por Ruíz Trejo, 2015). Es en este artículo donde abordó el efecto fotoeléctrico. El segundo artículo publicado el 11 de mayo de 2005, sobre el movimiento requerido por la teoría cinética molecular del calor de pequeñas partículas suspendidas en un líquido estacionario, estudió el movimiento browniano. El tercer artículo publicado el 30 de junio de 1905, Sobre la Electrodinámica de los Cuerpos en movimiento, es donde Einstein introdujo su teoría especial de la relatividad; rechazó la hipótesis de la existencia del éter luminífero: y donde postuló que la luz se propaga siempre con la velocidad c la cual es independiente del estado de movimiento del cuerpo emisor. Y el cuarto artículo publicado el 27 de septiembre de 1905, ¿Depende la masa inercial de la Energía?, es donde concluyó que, si un cuerpo emite energía $L$ en forma de radiación, su masa disminuye en la cantidad $L / \mathrm{c}^{2}$, un resultado mejor conocido como $\mathrm{E}=\mathrm{mc}^{2}$.

En el año 1921, cuando Albert Einstein era Profesor del Kaiser-Wilhelm-Institut (ahora Instituto Max Planck) de Física de Berlín, Alemania, recibió el Premio Nobel de Física "por sus servicios a la Física Teórica, y especialmente por su descubrimiento de la ley del efecto fotoeléctrico" (Nobelprize.org, 2015).

El efecto fotoeléctrico es el fenómeno por el cual electrones son emitidos desde la superficie de la materia (usualmente los metales) cuando la luz brilla sobre ellos. En el trabajo de Einstein de 1905 relacionado con la producción y la transformación de la luz, él explicó el efecto fotoeléctrico proponiendo que la luz consistía de pequeñas partículas llamadas cuantos o fotones por la teoría cuántica de Planck. Estos fotones llevan la energía que es proporcional a la frecuencia de la luz. Los electrones que en la materia absorben la energía del fotón son los que salen eyectados.

En su artículo Einstein reconoce la diferencia formal entre las concepciones teóricas que los físicos se han formado acerca de los gases y otros cuerpos ponderables, y la teoría de Maxwell de los procesos electromagnéticos del llamado espacio vacío.

"La teoría de onda de la luz, la cual opera con funciones espaciales continuas, se ha probado espléndidamente por ella misma al describir fenómenos puramente ópticos y probablemente nunca podrá ser reemplazada por otra teoría. Uno debe tener en mente, sin embargo, que las observaciones ópticas se aplican a tiempos promedios y no a valores momentáneos, y que es concebible que a pesar de la completa confirmación de las teorías de la 
difracción, reflexión, refracción, dispersión, etc., por experimentos, la teoría de la luz, la cual opera con funciones espaciales continuas, puede conducir a contradicciones con la experiencia cuando se la aplica al fenómeno de la producción y transformación de la luz.

Verdaderamente, me parece que las observaciones concernientes a la 'radiación del cuerpo negro', fotoluminiscencia, producción de rayos catódicos por luz, y otros grupos de fenómenos asociados con la producción o conversión de luz puede ser entendida mejor si uno asume que la energía de la luz está discontinuamente distribuida en el espacio. De acuerdo a la suposición a ser contemplada aquí, cuando un rayo de luz es dispersado desde un punto, la energía no es distribuida continuamente sobre el espacio siempre creciente, pero consiste de un número finito de cuantos de energía que están localizados en puntos en el espacio, se mueven sin dividirse, y pueden ser absorbidos o generados solamente como un todo.

... yo deseo comunicar mi tren de pensamientos y presento los hechos que me condujeron a este curso, en la esperanza que el punto de vista a ser elaborado pueda probar el uso para algunos investigadores en sus investigaciones." (Einstein, 1905, págs. 86-87)."

El efecto fotoeléctrico tiene muchas consecuencias importantes en nuestra vida diaria. Es la base para la fotosíntesis, los fotodiodos, se usa dentro de las fibras ópticas, las redes de telecomunicaciones, celdas solares, imágenes de varios tipos y muchas otras aplicaciones.

\section{5: ALBERT EINSTEIN}

El quinto hito en la historia de la luz tiene que ver con la incorporación de la luz en la cosmología mediante la teoría general de la relatividad de Einstein.

En 1905, Einstein publicó la Teoría Especial de la Relatividad fundamentada en dos postulados simples: 1) El principio de la relatividad especial: las leyes de la física propiamente establecidas deben ser independientes de la velocidad del observador, esto es, que el movimiento absoluto no puede ser detectado; 2) La velocidad de la luz en el espacio vacío es constante e independiente del estado de movimiento del emisor. La teoría se aplica a mediciones hechas en diferentes sistemas de referencia inercial que se mueven con velocidad constante unos con respecto a otros. 
En los años siguientes a 1905, Einstein continuó trabajando y profundizando sus estudios sobre la relatividad, desarrollando enfoques más avanzados. Así, en 1911 escribió sobre la gravitación y la propagación de la luz:

"En una memoria publicada hace cuatro años traté de responder a la pregunta de si la propagación de la luz está influida por la gravitación. Vuelvo a este tema porque mi presentación previa de la cuestión no me satisface; y por una razón más importante, porque ahora veo que una de las consecuencias más importantes de mi primer tratamiento puede ponerse a prueba experimentalmente. En efecto, de la teoría que aquí se expresa se sigue que los rayos de luz que pasan cerca del Sol son desviados por el campo gravitatorio de éste, de modo que la distancia angular entre el Sol y una estrella fija que parece próxima a él se incrementa aparentemente en casi un segundo de arco". (Einstein, 1911, pág. 898).

Pero fue en noviembre de 1915 cuando Albert Einstein, en cuatro históricas conferencias dictadas en la Academia de Ciencias Prusiana de Berlín, Alemania, presentó la nueva Teoría General de la Relatividad (Renn, 2015) (Kox, Klein, \& Schulmann, 1997). Entre los Artículos Colectados de Albert Einstein (Engel, 1997), para noviembre de 2015 aparecen los siguientes títulos: 1) Noviembre 4: Sobre la Teoría de la Relatividad (Einstein, 1915); 2) Sobre la Teoría General de la Relatividad (Addendum) (Einstein, 1915); 3) Noviembre 18: Explicación del Movimiento del Perihelio de Mercurio desde la Teoría General de la Relatividad (Einstein, 1915); 4) Noviembre 25: Las Ecuaciones de Campo de la Gravitación (Einstein, 1915).

La Teoría General de la Relatividad es el resultado de generalizar la Relatividad Especial a marcos de referencia no inerciales que se mueven con velocidades no constantes. La base de esta teoría es el Principio de Equivalencia que dice que: un campo gravitacional homogéneo es completamente equivalente a un marco de referencia uniformemente acelerado (Tipler \& Mosca, 2008). Einstein asumió que el principio de equivalencia se aplicaba a toda la física y no solamente a la mecánica. Con esto asumió que no hay experimento de ninguna clase que pueda distinguir movimiento uniformemente acelerado a partir de la presencia de un campo gravitacional.

La Teoría General de la Relatividad, además de introducir una manera diferente de pensar sobre la gravedad (en términos de la geometría), hace una serie de predicciones específicas observables que se desvían de la gravitación clásica Newtoniana, especialmente bajo fuertes campos gravitacionales (Kutner, 2007). 
Una de las principales consecuencias de la teoría general de la relatividad de acuerdo con la gravedad, es el efecto curvatura de la geometría del espaciotemporal. La teoría predice que los cuerpos dentro de un campo gravitatorio siguen una trayectoria espacial curva, aun cuando en realidad puedan estar moviéndose según las líneas del universo lo más rectas posibles a través de un espacio curvado.

Una manera de probar la relatividad general fue observando la curvatura de la luz de las estrellas pasando por el Sol. Esta deflexión fue bien observada el 29 de mayo de 1919 cuando el astrofísico británico Sir Arthur Eddington fotografió el eclipse total de Sol. En las fotografías, en el campo de visión cercano al Sol, imágenes de estrellas presentaron desplazamientos porque su luz fue curvada por el campo gravitacional del Sol. Este efecto solo puede observarse durante los eclipses solares, porque en otras condiciones el brillo del Sol no lo permite.

La relatividad general, "es ahora rutinariamente aceptada como el fundamento de nuestra descripción del universo en grande, lo que llamamos cosmología, de agujeros negros, de las estrellas de neutrones y de pequeñas correcciones de las orbitas de planetas y las naves espaciales en nuestro sistema solar" (Blandford, 2015).

Aunque los instrumentos no pueden ni ver ni medir el espacio temporal, varios son los fenómenos que han confirmado las predicciones de su curvatura (Taylor Reed, 2016). La evidencia experimental es grande, por ejemplo, las lentes gravitacionales. La luz se curva alrededor de un objeto masivo tal como un agujero negro, causando que actué como una lente para las cosas que están detrás de él. Este es el método que rutinariamente utilizan los astrónomos para estudiar las estrellas y las galaxias detrás de objetos masivos. Un ejemplo es la Cruz de Einstein, un cuásar en la constelación del Pegaso. El cuásar está aproximadamente a 8 billones de años luz de la Tierra, y tiene delante una galaxia que está alejada 400 millones de años luz. Cuatro imágenes del cuásar aparecen alrededor de la galaxia porque la intensa gravedad de la galaxia curva la luz que viene desde el cuásar.

Muchas son las confirmaciones de la relatividad general que provienen de las observaciones espaciales (Wall, 2015). La relatividad general predice que la luz curva su trayectoria alrededor de un cuerpo masivo como un cúmulo de galaxias, el cual curva significativamente el espacio temporal. Esto ha sido observado rutinariamente por los astrónomos cuando utilizan las lentes gravitaciones para estudiar fuentes de luz muy alejadas. En una escala más pequeña, estos fenómenos ayudan a los buscadores de exoplanetas, al estudiar como los sistemas estelares curvan la luz desde los objetos de fondo. 


\section{5: PENZÍAS Y WILSON}

En 1960, el Laboratorio de Telefonía Bell de Estados Unidos, en Holmdel, Nueva Jersey, construyó una antena para colectar y amplificar señales de radio y ser utilizada con el sistema satelital Echo. A los pocos años esta tecnología se volvió obsoleta. Con la antena en desuso, los científicos Arno Penzias y Robert Wilson, vieron la oportunidad de utilizarla para realizar experimentos y analizar señales de radio desde el espacio entre las galaxias. Ellos encontraron un ruido persistente que parecía venir de todas las direcciones del cielo, descartando que su fuente fuera La Vía Láctea, cualquier otra fuente extraterrestre, 0 aún la interferencia urbana de la vecina ciudad de Nueva York, o que fueran palomas metidas en la antena con forma de cuerno (Levine, 2009).

En julio de 1965, Penzias y Wilson divulgaron los resultados de sus observaciones en un documento titulado Una medición del exceso de Temperatura de Antena a 4080 Mc/s (Penzias \& Wilson, 1965, págs. 419-421). Aquí ellos reportaron que las mediciones de la temperatura efectiva del ruido en el cenit de una antena de cuerno-reflectora de 20 pies localizada en el Laboratorio de Crawford Hill, Holmdel, Nueva Jersey, a $4080 \mathrm{Mc} / \mathrm{c}$ dio un valor de $3.5^{\circ}$ más alto de lo esperado. Este exceso de temperatura, dentro de los límites de las observaciones realizadas, era isotrópico, no polarizado, y libre de observaciones estacionales (julio, 1964 - abril, 1965). La temperatura total de la antena medida en el cenit era de $6.7^{\circ} \mathrm{K}$ de los cuales, $2.3^{\circ} \mathrm{K}$ se debían a la absorción atmosférica, y $0.9^{\circ} \mathrm{K}$ a las contribuciones calculadas debidas a las perdidas óhmicas en la antena y la respuesta de retorno.

En este mismo documento Penzias y Wilson reportaron que, una posible explicación del exceso de temperatura de ruido observada era la que Dicke, Peebles, Roll, y Wilkinson daban en el documento titulado Radiación Cósmica de Cuerpo Negro (Dicke et al 1965, págs. 414-419) en la misma revista.

Pocos meses después, Penzias y Wilson publicaron otro artículo sobre las Mediciones de la Densidad de Flujo de Cas A at 4080 Mc/s (Penzias \& Wilson, 1965), a partir de valores que habían obtenido en seis noches de septiembre y octubre de 1964, utilizando la misma antena del Laboratorio de Crawford Hill. El valor obtenido fuede $1086 \times 10^{-23} \mathrm{~W} \mathrm{~m}^{-2}(\mathrm{c} / \mathrm{s})^{-1}$, con un error probable de aproximadamente 2 por ciento, basado en un 6.3 por ciento límite para 99 por ciento de nivel de confianza.

Desde hacía bastante tiempo se sabía que varios de los objetos astronómicos emitían radiación en forma de ondas de radio. La radioastronomía creció como un complemento importante de la astronomía óptica clásica. La radiación de ondas 
de radio se detectaba de varias maneras: las excitadas nubes de hidrogeno en la Galaxia radiaban, los electrones cósmicos moviéndose en espiral en los campos magnéticos débiles del espacio interestelar radiaban; las estrellas, galaxias y cuásares también se había encontrado que radiaban. Al estudiar todas estas fuentes, se hizo entonces necesario conocer su radiación, sobre la radiación general de fondo. La composición y el origen de esta radiación de fondo no fue bien entendida por un largo tiempo. (Nobelprize.org, 1978).

Cuando el descubrimiento de Penzias y Wilson se publicó, ya habían, sin embargo, varias especulaciones de la existencia de una débil radiación de fondo de microondas. El punto de partida de estas especulaciones fueron los intentos realizados desde los años 1940s, para explicar la síntesis de los elementos químicos. Una teoría fue desarrollada por el físico estadounidense George Gamow y su grupo sugiriendo que esta síntesis tuvo lugar en el principio de la existencia del universo.

Del estudio de los espectros de las estrellas y las galaxias se sabía que el universo se está expandiendo uniformemente. Esto significaba hasta cierto punto que, hace unos 15,000 millones de años el Universo era muy compacto, asumiéndose que fue creado por una explosión cósmica o "Big Bang", aunque otras explicaciones eran posibles. Esta teoría implicó que hace unos 10,000 millones de años la temperatura era muy alta, pudiendo dar lugar a reacciones nucleares que pudieron formar los elementos químicos, que se formaron de las partículas elementales asumidas que estaban presentes en el principio. Esto implicó una liberación de radiación desde la región de los rayos $X$, pasando por el visible, y las ondas de radio. Después de la hipotética explosión, la temperatura decreció rápidamente, surgiendo entonces la pregunta de, ¿dónde estaban los escombros de la explosión? La materia, consistente de hidrogeno, helio y otros elementos ligeros se habría condensado para formar las galaxias y las estrellas. Pero, qué pasaba con la radiación, era posible detectarla. La radiación que podría detectarse seria la correspondiente a un cuerpo con una temperatura de 3 grados sobre el cero absoluto. Pero era imposible detectar está débil radiación en el ruido cósmico.

Cuando Penzias y Wilson descubrieron la radiación cósmica de fondo de microondas era razonable pensar que esta era la radiación fósil del "Big Bang".

Para apoyar esta interpretación se realizaron muchas investigaciones para darle forma al espectro, el cual pronto mostró que correspondía a uno de una temperatura de 3 grados. Esto dio una evidencia sólida para apoyar el punto de vista de los restos de la radiación fósil del Big Bang. 
El descubrimiento de Penzias y Wilson fue fundamental. Hizo posible obtener información acerca de los procesos cósmicos que tuvieron lugar hace muchísimo tiempo, tanto como desde la creación del Universo.

En 1978 se les otorgó el Premio Nobel de Física a Arno Allan Penzias y a Robert Wood Woodrow Wilson, "por su descubrimiento de la radiación de microondas cósmica de fondo." Arno Allan Penzias, astrofísico, nació en Múnich, Alemania, el 26 de abril de 1933. Robert Woodrow Wilson, astrofísico, nació en Houston, Texas, Estados Unidos, el 10 de enero de 1936. Al momento de recibir el Premio Nobel, ambos trabajaban en el Laboratorio Bell, Holmdel, Nueva Jersey, Estados Unidos.

"La radiación cae hacia la tierra desde el espacio exterior. Esta radiación cósmica inicialmente parecía que era más débil a medida que la longitud de onda de la radiación era más corta. Sin embargo, cuando Penzias y Wilson estudiaron la radiación cósmica en 1964, ellos descubrieron que las microondas, con una longitud de onda de unos 7 centímetros era más fuerte de lo esperado... Esta radiación cósmica de fondo probablemente es un resto del Big Bang cuando se creó el Universo." (Nobelprize.org, 1978).

Así pues, el descubrimiento de la radiación cósmica de fondo de microondas hecha por Penzias y Wilson marcó otro hito en la historia de la ciencia de la luz.

\section{5: CHARLES KUEN KAO}

El séptimo hito en la historia de la ciencia de la luz se marcó por los logros alcanzados por Kao en la transmisión de luz por fibras para la comunicación óptica.

El uso de la fibra óptica fue un hecho que hizo posible superar las dificultades en la comunicación mundial a grandes distancias. Charles Kuen Kao fue el pionero que visualizó el uso de una guía de ondas de fibra óptica de un dieléctrico mono modal (vidrio) para la comunicación de largas distancias, una idea que en su tiempo parecía imposible. En noviembre de 1965, Kao y su colega George Hockham presentaron un artículo el cual hoy se reconoce como el principio de la revolución de la fibra óptica (Optical Fibre History, 2016). El artículo se tituló Guías de onda de superficie de fibra dieléctrica para frecuencias ópticas (Kao \& Hockham, 1966).

Hoy en día es posible recibir en pequeños y adecuados dispositivos, mensajes de texto, imágenes y videos que son enviados a casi la velocidad de la luz por fibras ópticas. Controlando la luz y dirigiéndola por guías de onda, mensajes codificados se pueden transmitir simultáneamente a muchas personas. 
Este desarrollo requirió de numerosos inventos, grandes y pequeños, los cuales forman los cimientos de la moderna sociedad de la información. La fibra óptica requirió de moderna tecnología del vidrio con el objeto de ser desarrollada y manufacturada. Una fuente confiable de luz también fue necesitada y esta fue proporcionada por la tecnología de los semiconductores. Finalmente, una ingeniosa red de trabajo también fue necesitada para ser ensamblada y extendida, consistente de transistores, amplificadores, interruptores, transmisores y receptores, así como otras unidades, todas trabajando juntas. La revolución de las telecomunicaciones fue hecha posible por el trabajo de miles de científicos e inventores de todas partes del mundo (The Royal Swedish Academy of Sciences, 2009).

El guiado de la luz en un medio utilizando el concepto de reflexión total interna es un asunto que fue discutido desde el siglo XIX; aunque se puede argumentar que fue conocido desde tiempos antiguos en Egipto y Mesopotamia. (Class for Physics of the Royal Swedish Academy of Sciences, 2009).

Las primeras ideas de aplicar luz guiada en pequeños rodos de vidrio datan desde los años 1920s. La transmisión de imágenes por medio de un manojo de estas fibras se utilizó en medicina (gastroscopio), en defensa (periscopio), y aún en la temprana televisión, aunque cuando las fibras se tocaban unas con otras o la superficie era rayada, la luz se filtraba y la fibra se desgastaba. La situación mejoró cuando a principios de los años 1950s, surgió la idea y la demostración que el revestimiento de las fibras ayudaba a la transmisión de la luz.

Para la comunicación a largas distancias, sin embargo, estas fibras de vidrio no eran efectivas y muy pocos fueron los que realmente se interesaron en la luz óptica. Aquellos eran los días de la electrónica y la radio tecnología. Pero el invento del láser a principios de los 1960 fue un paso decisivo para las fibras ópticas. El láser era una fuente de luz intensa y altamente enfocada que podía ser bombardeada en la fibra óptica delgada. La información podía ser codificada en unos y ceros y enviada en la rápida luz. Sin embargo, un gran problema era la transmisión por largas distancias, en 20 metros solo el 1 por ciento de la luz que entraba en la fibra óptica permanecía.

Reducir esta pérdida de luz llegó a ser un reto para Charles Kuen Kao. Para ese tiempo él ya era empleado de los Standard Telecommunication Laboratories. Allí él estudio meticulosamente las fibras de vidrio junto con su joven colega George A. Hockman. Su meta era que al menos $1 \%$ de la luz que entrara a una fibra de vidrio pudiera permanecer después de haber viajado 1 kilómetro. 
En 1966, Kao presentó sus conclusiones. El resultado más importante era que las pérdidas en el medio dieléctrico eran causadas principalmente por la absorción y la dispersión, esta última causada por impurezas en particular de iones de hierro. Fibras con vidrios de mayor pureza podían ser buenas candidatas para las comunicaciones ópticas. Las fibras de modo simple eran el mejor medio de transmisión para las comunicaciones ópticas. Las operaciones en multi modo resultan en mayores pérdidas y ampliación temporal debido a dispersiones más altas. Kao concluyó con una frase visionaria (Class for Physics of the Royal Swedish Academy of Sciences, 2009): "[...] una fibra de material vidrioso construida en una estructura revestida [...] representa una posible guía de onda óptica práctica con potencial importante como una nueva forma de medio de comunicación [...] Comparado con el cable coaxial existente y los sistemas de radio, esta forma de guía de onda tiene una mayor capacidad de información y posiblemente ventajas en los costos de materiales básicos." (Kao \& Hockham, 1966). El entusiasmo de Kao inspiró a otros investigadores a compartir su visión del futuro potencial de la fibra óptica (The Royal Swedish Academy of Sciences, 2009).

El problema por resolver era la fabricación de fibras ópticas sin impurezas. Cuatro años después del artículo de Kao y Hockham, un equipo de la Corning Glass Works de Estados Unidos tuvo éxito en hacer las fibras ópticas que Kao había divisado. A los pocos años, en varios países, se utilizaron varias tecnologías que permitieron la atenuación de las pérdidas a menos de $1 \mathrm{~dB} / \mathrm{km}$ lo cual era mucho más del umbral fijado por Kao. Hoy, la atenuación de la luz a una longitud de onda de 1.55 $\mu \mathrm{m}$ en una fibra es menos de $0.2 \mathrm{~dB} / \mathrm{km}$. Una fibra óptica moderna es un medio extraordinariamente transparente, con más del $95 \%$ de luz propagada después de $1 \mathrm{~km}$.

Charles K. Kao nació el 4 de noviembre de 1933 en Shanghai, China. Recibió el Premio Nobel de Física en 2009. Al momento de otorgársele el premio estaba afiliado a los Standard Telecommunication Laboratories, Harlow, Inglaterra, y la Universidad China de Hong Kong, en Hong Kong, China (Nobelprize.org, 2009).

Los logros alcanzados por Kao en la transmisión de luz por fibras para la comunicación óptica, no hay duda que son otro hito en la historia de la ciencia de la luz. 


\section{CONSIDERACIONES FINALES}

La celebración en 2015 del Año Internacional de la Luz ha sido una magnífica oportunidad para celebrar los aniversarios de los siete hitos de la historia de la ciencia de la luz presentados; así como para resaltar además por medio de los descubrimientos científicos señalados, la importancia de la enseñanza de las ciencias en nuestro país.

Oportuno es entonces cerrar este documento, con algunos fragmentos de la Resolución Declaratoria del 2015 Año Internacional de la Luz:

"Considerando que las aplicaciones de la ciencia y la tecnología de la luz son esenciales para los avances ya alcanzados y futuros en las esferas de la medicina, la energía, la información y las comunicaciones, la fibra óptica, la agricultura, la minería, la astronomía, la arquitectura, la arqueología, el ocio, el arte y la cultura, entre otras, así como en muchos otros sectores industriales y servicios, y que las tecnologías basadas en la luz contribuyen al logro de las metas de desarrollo convenidas internacionalmente, entre otras cosas al proporcionar acceso a la información y aumentar la salud y el bienestar de la sociedad,

Considerando también que la tecnología y el diseño pueden desempeñar un papel importante en el logro de una mayor eficiencia energética, en particular al limitar el despilfarro de energía, y en la reducción de la contaminación lumínica, que es fundamental para la conservación de cielos oscuros,

Considerando que la celebración de los aniversarios de estos descubrimientos en 2015 ofrecería una importante oportunidad para destacar la continuidad de los descubrimientos científicos en diferentes contextos, haciendo especial hincapié en el empoderamiento de las mujeres en el ámbito científico y la promoción de la enseñanza de las ciencias entre los jóvenes, especialmente en los países en desarrollo,

1. Decide proclamar el año 2015 Año Internacional de la Luz y las Tecnologías Basadas en la Luz;

4. Alienta a todos los Estados, al sistema de las Naciones Unidas y a todos los demás agentes a que aprovechen el Año Internacional para promover 
medidas a todos los niveles, incluso mediante la cooperación internacional, y aumenten la conciencia del público sobre la importancia de las ciencias de la luz, la óptica y las tecnologías basadas en la luz y de promover un amplio acceso a los nuevos conocimientos y actividades conexas. (Organización de las Naciones Unidas, 2014 ).

\section{Agradecimientos.}

Agradezco al Nodo Internacional de Honduras para la Celebración del 2015 Año Internacional de la Luz, por brindarme la oportunidad de presentar este trabajo en la Conferencia Inaugural del año de celebraciones, en la Facultad de Ciencias Espaciales de la Universidad Nacional Autónoma de Honduras.

\section{REFERENCIAS BIBLIOGRÁFICAS}

- Alonso Sanchez, M. 2015, abril 5. Debate histórico sobre la naturaleza de la luz. Obtenido de Debate histórico sobre la naturaleza de la luz: http://intercentres. edu.gva.es/iesleonardodavinci/Fisica/Luz/Naturaleza-luz.pdf

- Bachiller,R.015, septiembre16.jLaluzesunaonda!ObtenidodeEIMundo.Ciencia: http://www.elmundo.es/ciencia/2015/09/16/55f678c3ca4741ce708b4570.html

- Beléndez, A.2015, abril 22. Young, Fresnel y la teoría ondulatoria de la luz. Obtenido de Física para tod@s:http://blogs.ua.es/fisicateleco/2015/04/youngfresnel/

- Blandford, R. D.2015. A century of general relativity: Astrophysics and cosmology. Science, 1103-1108.

- Calvo, M. L. 2015. La excepcional contribución de Ibh-al-Hytham (Alhacén) a las ciencias. Revista de Física, 29 - 1 (Enero-marzo 2015), 31-35.

- $\quad$ Class for Physics of the Royal Swedish Academy of Sciences.2009, octubre 6. Two Revolutionary Optical Technologies. Obtenido de Scientific Background on the Nobel Prize in Physics 2009: http://www.nobelprize.org/nobel_prizes/ physics/laureates/2009/advanced.html 
- Dicke, R. H., Peebles, P. J., Roll, P. J., \& Wilkinson, D. T. 1965, julio. Cosmic Black-Body Radiation. Astrophysical Journal Letters, 142(1), 414-419. doi:10:1086/14806

- Eckem, U. 2005, febrero 19. Annalen der Physik (AdP) \& Albert Einstein. Obtenido de Einstein in AdP: http://myweb.rz.uni-augsburg.de/ eckern/adp/ history/Einstein-in-AdP.htm

- Einstein, A.

1905. Zur Elektrodynamik bewegter Körper. Annalen der Physik, 891-921.

1911. Uber den Einfluß der Schwerkraft auf die Ausbreitung des Lichtes. Annalen der Physik, 35, 98-908.

1915, noviembre 4. On the General Theory of Relativity. Obtenido de Doc. 21 General Relativity: http://einsteinpapers.press.princeton.edu/vol6trans/110 1

1915, noviembre. On the General Theory of Relativity (Addendum). Obtenido de Doc. 22 Addendum to Doc. 21: http://einsteinpapers.press. princeton.edu/vol6-trans/120

1915, noviembre 18. Explanation of the Perihelion Motion of Mercury from the General Theory of Relativity. Obtenido de Volume 6: The Berlin Years: Writings, 1914-1917: http://einsteinpapers. press. princeton.edu/vol6doc/261

1915, noviembre 25. The Field Equations of Gravitation. Obtenido de Doc. 25 Field Equations of Gravitation: http://einsteinpapers.press.princeton. edu/vol6-trans/129

- Engel, A.1997, mayo . Volume 6: The Berlin Years: Writings, 1914-1917 (English translation supplement). (Princeton University Press) Obtenido de The Collected Papers of Albert Einstein:http://einsteinpapers.press.princeton.edu/ vol6-trans

- González-Cano, A. 2015. Alhacén: una revolución óptica. ARBOR Ciencia, Pensamiento y Cultura, 191-775 (septiembre- octubre 2015, a262), 1-13. doi:http://dxdx.doi.org/10.3989/arbor.2015.775n5001 
- Hecht, E., \& Zajac, A.1986. OPTICA. Wilmington: Addison-Wesley Iberoamericana, S. A.

- Kao, K. C., \& Hockham, G. A. 1966, julio. Dielectric-fibre surface waveguides por optical frequencies. Institution of ElectricalEngineers Proceedengs $\mathrm{J}$ Optoelectronics, 113(7), 1151-1158. doi:10.1049/piee.1966.0189

- Kox, A. J., Klein, M. J., \& Schulmann, R.1997. The Collected Papers of Albert Einstein. The Berlin Years: Writings, 1914-1917. Princeton: Princeton University Press.

- Kutner, M. L. 2007. Astronomy, A Physical Perspective . Cambridge: Cambridge University Press.

- Levine, A. G. 2009. Arno Penzias y Robert Wilson, Bell Labs, Holmdel, N.J. Obtenido de The Large Horn Antenna and the Discovery of Cosmis Mlcrowave Background Radiation: https://www.aps.org/programs/outreach/history/ historicsites/penziaswilson.cfm

- Maxwell, J. C. 1865. A Dynamical Theory of the Electromagnetic Field. Phylosophycal Transactions, 459-512. Obtenido de http://rstl. royalsocietypublishing.org/content/155/459.full.pdf+html

- Nobel Lectures. 1967. Physics 1901-1921. Amsterdam: Elsevier Publishing Companu.

- Nobelprize.org.

1978, octubre 17. The Nobel Prize in Physics

1978. Obtenido de Press Release: https://www.nobelprize.org/nobel_ prizes/physics/laureates/1978/press.html

2009. The Nobel Prize in Physics 2009. Obtenido de Charles K. Kao Facts: http://www.nobelprize.org/nobel_prizes/physics/laureates/

2009/kao-facts.html2015, abril 4. The Nobel Prize in Physics 1921, Albert Einstein. Obtenido de Albert Einstein Facts: https://www.nobelprize.org/ nobel_prizes/physics/laureates/1921/einstein-facts.html

- Optical Fibre History. 2016. Optical Fibre History. Obtenido de How the fibre communications revolution began:http://opticalfibrehistory.co.uk/ 
- Organización de las Naciones Unidas. 2014 . 68/21. Año Internacional de la Luz y las Tecnologías Basadas en la Luz, 2015. New York: Asamblea General.

- Penzias, A. A., \& Wilson, R. W.

1965, julio. A measurement of excess Antenna Temperatura at $4080 \mathrm{Mc} / \mathrm{s}$. Astrophysical Journal Letters, 142(1), 419-421. doi:10.1086/148307

1965, octubre. Measurements of the Flux Density of CAS at $4080 \mathrm{Mc} / \mathrm{s}$. Astrophysical Journal, 142(3), 149-1155. doi:10.1086/148384

- Renn, J. 2015, abril 8. Einstein's path to General Relativity. Obtenido de Max Planck Institute for History of Science: http://www.ihes.fr/ vanhove/Slides/rennIHES-mars 2013.pdf

- Ruíz Trejo, E. 2015, marzo 1. Sobre un punto de vista heurístico concerniente a la producción y transferencia de la luz. Obtenido de Einstein 1905: http:// casanchi.com/fis/einstein1905/uno/uno_e.pdf

- Sabra, A. I. 1989. The Optics of Ibn al-Haytham [Alhacén] (Vols. Books I-III). London: The Warbug Institute.

- Taylor Reed, N. 2016, julio 12. Einsteoin's Theory of General Relativity. Obtenido de Space.com: http://www.space.com/17661-theory-general-relativity.html

- The Royal Swedish Academy of Sciences. 2009. The Masters of Light. Obtenido de The Nobel Prize in Physics 2009: http://www.nobelprize.org/nobel_prizes/ physics/laureates/2009/popular.html

- Tipler, P. A., \& Mosca, G. 2008. Física con Física Moderna, para científicos e ingenieros (Sexta ed.). New York: W. H. Freeman and Company.

- Wall, M. 2015, marzo 5. General Relativity at 100: Einstein's Famous Theory Has Aged Well. Obtenido de Sapace.com > Science \& Astronomy: http://www. space.com/28741-einstein-general-relativity-100-years.html 
- Wikipedia.

2015, abril 5. Alhacén. Obtenido de Wikipedia. La enciclopedia libre: https:// es.wikipedia.org/wiki/Alhac\%C3\%A9n

2015, abril 5. Augustin Fresnel. Obtenido de Augustin Fresnel: https:// es.wikipedia.org/wiki/Augustin_Fresnel

2015, abril 5. James Clerk Maxwell. Obtenido de James Clerk Maxwell: https://es.wikipedia.org/wiki/James_Clerk_Maxwell 